\title{
Joint Semiblind Frequency Offset and Channel Estimation for Multiuser MIMO-OFDM Uplink
}

\author{
Yonghong Zeng, Senior Member, IEEE, A. Rahim Leyman, and Tung-Sang Ng, Fellow, IEEE
}

\begin{abstract}
A semiblind method is proposed for simultaneously estimating the carrier frequency offsets (CFOs) and channels of an uplink multiuser multiple-input multiple-output orthogonal frequency-division multiplexing (MIMO-OFDM) system. By incorporating the CFOs into the transmitted symbols and channels, the MIMO-OFDM with CFO is remodeled into an MIMO-OFDM without CFO. The known blind method for channel estimation (Zeng and $\mathrm{Ng}$ in 2004) (Y. H. Zeng and T. S. Ng, "A semi-blind channel estimation method for multi-user multi-antenna OFDM systems," IEEE Trans. Signal Process., vol. 52, no. 5, pp. 14191429, May 2004.) is then directly used for the remodeled system to obtain the shaped channels with an ambiguity matrix. A pilot OFDM block for each user is then exploited to resolve the CFOs and the ambiguity matrix. Two dedicated pilot designs, periodical and consecutive pilots, are discussed. Based on each pilot design and the estimated shaped channels, two methods are proposed to estimate the CFOs. As a result, based on the second-order statistics (SOS) of the received signal and one pilot OFDM block, the CFOs and channels are found simultaneously. Finally, a fast equalization method is given to recover the signals corrupted by the CFOs.
\end{abstract}

Index Terms-Carrier frequency offset (CFO), channel estimation, frequencyoffset, multiple-input multiple-output (MIMO), multiuser, orthogonal frequency-division multiplexing (OFDM), semiblind, subspace method, zero padding.

\section{INTRODUCTION}

$\mathbf{T}$ HE COMBINATION of multiple input multiple output (MIMO) and orthogonal frequency-division multiplexing (OFDM), that is, the MIMO-OFDM, has emerged as a major candidate for the future fourth-generation (4G) communications [2]. It is well known that OFDM is vulnerable to carrier frequency offset (CFO) caused by transceiver mismatch and/or Doppler shift. Due to the nature of multiple transmitters and receivers in the MIMO-OFDM, frequency synchronization and channel estimation become more challenging. In MIMO-

Paper approved by Y. Li, the Editor for Wireless Communication Theory of the IEEE Communications Society. Manuscript received July 12, 2005; revised January 25, 2006. This work was in part supported by the Agency for Science, Technology and Research (A*STAR) Embedded and Hybrid Systems (EHS) Research, Singapore, under Grant 0221060041 . This paper was presented in part at the 16th IEEE Annual International Symposium on Personal Indoor and Mobile Radio Communications, 2005.

Y. H. Zeng is with the Institute for Infocomm Research, Agency for Science, Technology and Research (A*STAR), Singapore 119613, Singapore (e-mail: yhzeng@ieee.org).

A. R. Leyman is with the Institute for Infocomm Research, Agency for Science, Technology and Research (A*STAR), Singapore 119613, Singapore. $\mathrm{He}$ is also with the Department of Electrical Computer Engineering, National University of Singapore, Singapore 119275, Singapore (e-mail: larahim@i2r.astar.edu.sg).

T. S. Ng is with the University of Hong Kong, Hong Kong (e-mail: tsng@eee.hku.hk).

Digital Object Identifier 10.1109/TCOMM.2007.910637
OFDM, different transmitter-receiver pair may have a different $\mathrm{CFO}$, that is, there may be multiple CFOs required to be estimated. It is a challenging problem to resolve the multiple CFOs in a multiuser uplink system with unknown multipath channels [3]. For the channel estimation, since the number of channels increases rapidly with the number of transmitters and receivers, more pilot inputs and new methods are needed.

Although there have been quite a few CFO estimation methods for OFDM [3]-[14], most of them are applicable only to single-input single-output (SISO) OFDM systems [4]-[11]. Most of the researches of CFO estimation for the MIMO-OFDM assume that only single-frequency offset exists between the transmit and the receive antennas [12], [13], which is unrealistic in most cases for the uplink. Little research work has been done for estimating the multiple CFOs in the MIMO uplink (with or without OFDM modulation) [15], [16] (flat-fading channel model is assumed in the two papers). Although there are some researches on the multiple CFOs estimation for orthogonal frequency-division multiple access (OFDMA) uplink (also called multiuser OFDM) [3], [14], the methods cannot be used for the MIMO-OFDM uplink, because the structures of the two systems are very different. Furthermore, most known research works deal with CFO and channel-estimation separately. In fact, almost all known channel estimation methods for MIMO-OFDM assume that the CFOs have been acquired before resolving the channels [1], [17]-[20], and most CFO estimation methods for MIMO uplink (or OFDMA uplink) do not consider channel estimation at all [3], [12]-[15]. A blind method is proposed in [8] for the joint estimation of the CFO and the channel, but it is only applicable to SISO-OFDM. A maximum likelihood estimator is proposed in [16] for the channels and CFOs of the MIMO uplink, but a flat-fading channel model is assumed.

In this paper, we consider the uplink MIMO-OFDM assuming that every transmitter has its own RF device and transmits its own data independently at the same time and same frequency (this is different from the OFDMA [14]). This happens in two situations: 1) the input data stream is divided into multiple data streams and transmitted by multiple antennas at the same time and same frequency band [like the very-high-data-rate blocked asmchronous transmission (V-BLAST)] [21], [22] and 2) different users at different locations transmit their data at the same time and same frequency [1], [23] [also called spatial division multiple access (SDMA)]. The two cases can be treated as the same if we define an independent data stream as an user (virtual user). At the base station, the number of receiving antennas must be larger than or equal to the number of users (transmitting antennas) in order to recover the transmitted signals. It is 
reasonable to assume that all the antennas in the base station share the same oscillator. We assume that the channels are frequency selective with multipath, but static within several OFDM blocks. Under this assumption, the CFOs can be incorporated into the transmitted symbols and channels. Hence, the MIMOOFDM with CFOs can be remodeled into a MIMO-OFDM without CFO (but the channels and transmitted symbols are changed). The known blind method for channel estimation [1] is, then, directly used for the remodeled system to obtain an estimation for the CFO incorporated channels with an ambiguity matrix. A pilot OFDM block for each user is then exploited to resolve the CFOs and the ambiguity matrix. Two dedicated pilot designs, periodical and consecutive pilots, are discussed. Based on each pilot design and the estimated shaped channels, two methods are proposed to estimate the CFOs. As a result, based on the second-order statistics (SOS) of the received signal and one pilot OFDM block, the CFOs and channels are found simultaneously.

A major advantage of an OFDM is that it has the socalled "per-ton" equalization based on the fast Fourier transform (FFT). If no CFOs exist, this advantage is kept in the MIMOOFDM, as shown in [1] and [2]. However, the CFOs complicate the problem. The CFOs destroy the orthogonality among the subcarriers and introduce intercarrier interferences (ICI). Therefore, the per-tone equalization cannot be used anymore. Usually, there are two ways to handle this problem [24]. The first one is feedbacking the estimated CFOs at the base station to the users and asking them to adjust their oscillator. Thereafter, the received signals are $\mathrm{CFO}$ free. This method needs a feedback channel and may not be viable sometimes. Furthermore, if the CFO is caused by Doppler shift or random errors, it is impossible to adjust at the transmitter. The second one is separating the ICI corrupted signal at the base station based on the estimated CFOs. The problem with this way is that the separation may be rather complicated [24]. Here we consider the second way. A method is proposed to recover the signals based on FFT. The computational complexity is only moderately higher than that of the "per-tone" equalization [1].

The rest of the paper is organized as follows. In Section II, we present the uplink MIMO-OFDM system and remodel it. The blind estimation of the modified channel is discussed in Section III. Section IV presents two pilot designs to resolve the CFOs and the ambiguity. The equalization method is presented in Section V. Some simulation results and discussions are provided in Section VI. Finally, conclusion is drawn in Section VII.

In the following, superscripts $T, \dagger$, and $*$ stand for transpose, Hermitian, and conjugate, respectively.

\section{UPLINK MIMO-OFDM AND REMODELING}

The multiuser MIMO-OFDM uplink system is shown in Fig. 1. Assume that there are $K$ users who share the same time and frequency band, and $J$ omnidirectional receiving antennas at the base station. Each antenna can receive signals from every user in the cell by using the base station. We assume that each user uses the zero-padding OFDM (ZP-OFDM) [25]-[27] instead of the cyclic prefix OFDM (CP-OFDM), because ZP-

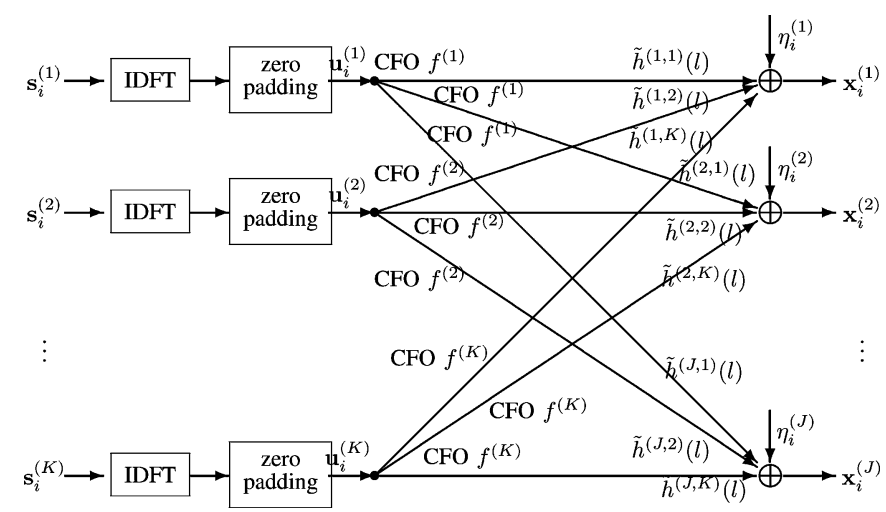

Fig. 1. Multiuser MIMO-OFDM system (uplink).

OFDM avoids interblock interference (IBI), and therefore, simplifies channel estimation and equalization [25]-[27]. In the ZPOFDM, the symbols to be transmitted are grouped into blocks with each block having $N$ symbols, each block is transformed by the inverse discrete Fourier transform (IDFT), and then, $L$ $(L \leq N)$ zeros are added to the tail of each transformed block (zero padding), where cyclic prefix is no longer needed. Each user transmits its OFDM modulated signal. Let $\mathbf{s}_{i}^{(k)}$ be the block symbol to be transmitted by user $k$ at time $i$ (before OFDM modulation), where

$$
\begin{aligned}
\mathbf{s}_{i}^{(k)}=\left(s_{i}^{(k)}(0), s_{i}^{(k)}(1), \ldots, s_{i}^{(k)}(N-1)\right)^{T} & \\
& k=1,2, \cdots, K, i=0,1, \ldots
\end{aligned}
$$

and its IDFT is $\mathbf{u}_{i}^{(k)} \cdot \mathbf{u}_{i}^{(k)}$ is zero padded with $L$ zeros and then transmitted. Let $\tilde{h}^{(j, k)}(l)\left(l=0,1, \ldots, L_{j, k}\right)$ be the multipath channel response (including the transmitting and receiving filters) from user $k$ to antenna $j$, where $L_{j, k}$ is the channel order. In general, there is a carrier frequency mismatch between the user $k$ and antenna $j$, which causes a CFO $f^{(j, k)}$. For uplink, it is reasonable to assume that all the antennas at the base station share the same oscillator. Hence, $f^{(j, k)}=f^{(k)}$ for all $j$. Then, if $f^{(k)}$ is normalized by subcarrier separation and the CFOs and channels are invariant within several OFDM blocks, the received $i$ th block at antenna $j$ is

$$
\begin{aligned}
x_{i}^{(j)}(n)= & \sum_{k=1}^{K} \omega_{N}^{f^{(k)}(n+i M)} \\
& \cdot \sum_{l=0}^{L_{j, k}} \tilde{h}^{(j, k)}(l) u_{i}^{(k)}\left(n-l-\tau_{j, k}\right)+\eta_{i}^{(j)}(n) \\
= & \sum_{k=1}^{K} \omega_{N}^{f^{(k)}(n+i M)} \\
& \cdot \sum_{l=\tau_{j, k}}^{L_{j, k}+\tau_{j, k}} \tilde{h}^{(j, k)}\left(l-\tau_{j, k}\right) u_{i}^{(k)}(n-l)+\eta_{i}^{(j)}(n), \\
n= & 0,1, \ldots, M-1
\end{aligned}
$$

where $M=N+L, \omega_{N}=e^{j 2 \pi / N}(\jmath=\sqrt{-1}), \tau_{j, k}$ is the relative time delay of user $k$ to antenna $j$ (for an asynchronous 
system), and $\eta_{i}^{(j)}(n)$ is the channel noise. Note that $u_{i}^{(k)}(n)=0$, if $n<0$ or $N \leq n<M$. If the $\mathbf{Z P}$ length $L$ is large enough such that $L_{j, k}+\tau_{j, k} \leq L$, then (1) can be rewritten as

$$
\begin{aligned}
x_{i}^{(j)}(n)= & \sum_{k=1}^{K} \omega_{N}^{f^{(k)}(n+i M)} \sum_{l=0}^{L} h^{(j, k)}(l) u_{i}^{(k)}(n-l) \\
& +\boldsymbol{\eta}_{i}^{(j)}(n), \quad n=0,1, \ldots, M-1
\end{aligned}
$$

where $h^{(j, k)}(l)=\tilde{h}^{(j, k)}\left(l-\tau_{j, k}\right)$, and $h^{(j, k)}(l)$ is set to zero for $l<\tau_{j, k}$ or $l>L_{j, k}+\tau_{j, k}\left(h^{(j, k)}(l)\right.$ is the effective channel of the system). Please note that here the time delays have been incorporated into the channels.

We assume that $J \geq K$ (this is necessary in order to recover the transmitted signals). Defining

$$
\begin{aligned}
\hat{h}^{(j, k)}(l) & =\omega_{N}^{f^{(k)} l} h^{(j, k)}(l) \\
\hat{u}_{i}^{(k)}(n) & =\omega_{N}^{f^{(k)}(n+i M)} u_{i}^{(k)}(n)
\end{aligned}
$$

we turn (2) into

$$
x_{i}^{(j)}(n)=\sum_{k=1}^{K} \sum_{l=0}^{L} \hat{h}^{(j, k)}(l) \hat{u}_{i}^{(k)}(n-l)+\eta_{i}^{(j)}(n) .
$$

In (4), the CFOs are embedded into the channels and transmitted symbols. Therefore, the semiblind method [1] for estimating the channels of the MIMO-OFDM system without CFO can be directly used here. Let

$$
\begin{aligned}
& \hat{\mathbf{u}}_{i}=\left[\hat{u}_{i}^{(1)}(0) \cdots \hat{u}_{i}^{(K)}(0) \cdots\right. \\
& \left.\hat{u}_{i}^{(1)}(N-1) \cdots \hat{u}_{i}^{(K)}(N-1)\right]^{T} \\
& \mathbf{x}_{i}=\left[x_{i}^{(1)}(0) \cdots x_{i}^{(J)}(0) \cdots\right. \\
& \left.x_{i}^{(1)}(M-1) \cdots x_{i}^{(J)}(M-1)\right]^{T} \\
& \boldsymbol{\eta}_{i}=\left[\eta_{i}^{(1)}(0) \cdots \eta_{i}^{(J)}(0) \cdots\right. \\
& \left.\eta_{i}^{(1)}(M-1) \cdots \eta_{i}^{(J)}(M-1)\right]^{T} \\
& \hat{\mathbf{h}}(l)=\left[\begin{array}{ccccc}
\hat{h}^{(1,1)}(l) & \hat{h}^{(1,2)}(l) & \ldots & \ldots & \hat{h}^{(1, K)}(l) \\
\hat{h}^{(2,1)}(l) & \hat{h}^{(2,2)}(l) & \ldots & \ldots & \hat{h}^{(2, K)}(l) \\
\vdots & \vdots & & & \vdots \\
\hat{h}^{(J, 1)}(l) & \hat{h}^{(J, 2)}(l) & \ldots & \ldots & \hat{h}^{(J, K)}(l)
\end{array}\right] \\
& \hat{\mathbf{H}}=\left[\begin{array}{ccccc}
\hat{\mathbf{h}}(0) & & & & \\
\vdots & \hat{\mathbf{h}}(0) & & & \\
\hat{\mathbf{h}}(L) & \vdots & \ddots & & \\
& \hat{\mathbf{h}}(L) & & \ddots & \\
& & \ddots & & \hat{\mathbf{h}}(0) \\
& & & \ddots & \vdots \\
& & & & \hat{\mathbf{h}}(L)
\end{array}\right]
\end{aligned}
$$

where $\hat{\mathbf{H}}$ is a $J M \times K N$ block lower triangular Toeplitz matrix with the first $J M \times K$ block column being

$$
\left[\hat{\mathbf{h}}^{T}(0), \hat{\mathbf{h}}^{T}(1), \ldots, \hat{\mathbf{h}}^{T}(L), 0, \ldots, 0\right]^{T} .
$$

Then, (4) is turned to a matrix form as

$$
\mathbf{x}_{i}=\hat{\mathbf{H}} \hat{\mathbf{u}}_{i}+\boldsymbol{\eta}_{i}, \quad i=0,1, \ldots
$$

\section{Blind Estimation OF THE MODIFIED CHANNELS}

To use the blind subspace method, we need the following assumptions for the statistical properties of transmitted symbols $s_{l}^{(k)}(n)$ and channel noise $\eta_{i}^{(j)}(n)$.

(A1) Noises are white and uncorrelated, that is

$$
\mathrm{E}\left(\eta_{i}^{(j)}(n)\left(\eta_{l}^{(k)}(m)\right)^{*}\right)=\left\{\begin{array}{cc}
\sigma_{\eta}^{2}, & (i, j, n)=(l, k, m) \\
0, & (i, j, n) \neq(l, k, m) .
\end{array}\right.
$$

(A2) Noises and transmitted signals are uncorrelated, that is

$$
\mathrm{E}\left(\eta_{i}^{(j)}(n)\left(s_{l}^{(k)}(m)\right)^{*}\right)=0 .
$$

Here, $\mathrm{E}(y)$ means the mathematical expectation of a random variable $y$.

Based on the model (4) or (10), we can directly use the blind subspace method in [1] to find an estimation for the modified channels $\hat{\mathbf{h}}(l)$ with ambiguity. For easy reading, we summarize the algorithm in the following.

Algorithm 1: Blind estimation of the modified channels.

Step 1. Compute $\mathbf{R}_{x}=\mathrm{E}\left(\mathbf{x}_{i} \mathbf{x}_{i}^{\dagger}\right) \approx \frac{1}{N_{s}} \sum_{i=1}^{N_{s}} \mathbf{x}_{i} \mathbf{x}_{i}^{\dagger}$, where $N_{s}$ is the number of output block samples used for computing the statistics.

Step 2. Find $q=J M-K N$ coorthogonal eigenvectors, $\boldsymbol{\beta}_{l}=\left(\boldsymbol{\beta}_{l}^{T}(0), \boldsymbol{\beta}_{l}^{T}(1), \ldots, \boldsymbol{\beta}_{l}^{T}(M-1)\right)^{T}(l=0,1, \ldots, q-1)$, corresponding to the smallest $q$ eigenvalues of matrix $\mathbf{R}_{x}$, where $\boldsymbol{\beta}_{l}(m)$ is a vector of length $J$.

Step 3. Form the matrix $\mathbf{G}$ from $\boldsymbol{\beta}_{l}$, and compute the eigenvalue decomposition (EVD) of $\mathbf{G}$, where

$$
\mathbf{G}=\sum_{l=0}^{q-1} \mathbf{G}_{l}^{\dagger} \mathbf{G}_{l}
$$

and

$$
\mathbf{G}_{l}=\left[\begin{array}{cccc}
\boldsymbol{\beta}_{l}^{\dagger}(L) & \boldsymbol{\beta}_{l}^{\dagger}(L-1) & \cdots & \boldsymbol{\beta}_{l}^{\dagger}(0) \\
\boldsymbol{\beta}_{l}^{\dagger}(L+1) & \boldsymbol{\beta}_{l}^{\dagger}(L) & \cdots & \boldsymbol{\beta}_{l}^{\dagger}(1) \\
\vdots & \vdots & & \vdots \\
\boldsymbol{\beta}_{l}^{\dagger}(M-1) & \boldsymbol{\beta}_{l}^{\dagger}(M-2) & \cdots & \boldsymbol{\beta}_{l}^{\dagger}(N-1)
\end{array}\right] .
$$

Choose $K$ eigenvectors corresponding to the $K$ smallest eigenvalues respectively to form the columns of matrix $\mathbf{F}$.

Step 4. Let $\overline{\mathbf{h}}(l)$ denotes the submatrix of $\mathbf{F}$ [the $l J$ to $(l+$ 1) $J-1$ rows of $\mathbf{F}], l=0,1, \ldots, L$. Then, $\overline{\mathbf{h}}(l)$ is the modified channel with ambiguity.

Based on the solution obtained from the subspace method, we know that there is an unknown constant invertible matrix $\mathbf{b}$ such that $\hat{\mathbf{h}}(l)=\overline{\mathbf{h}}(l) \mathbf{b}$. Let $\overline{\mathbf{H}}$ be the matrix in the same form 
as $\hat{\mathbf{H}}$ with $\hat{\mathbf{h}}(l)$ replaced by $\overline{\mathbf{h}}(l)$, and define

$$
\begin{gathered}
\mathbf{B}=\operatorname{diag}(\mathbf{b}, \mathbf{b}, \ldots, \mathbf{b}), \\
\mathbf{D}_{i}(n)=\operatorname{diag}\left(\omega_{N}^{f^{(1)}(n+i M)} \omega_{N}^{f^{(2)}(n+i M)}, \ldots,\right. \\
\left.\omega_{N}^{f^{(K)}(n+i M)}\right), n=0,1, \ldots, N-1, \\
\mathbf{D}_{i}=\operatorname{diag}\left(\mathbf{D}_{i}(0), \mathbf{D}_{i}(1), \ldots, \mathbf{D}_{i}(N-1)\right), \\
\mathbf{u}_{i}=\left[u_{i}^{(1)}(0), \ldots, u_{i}^{(K)}(0), \ldots,\right. \\
\left.u_{i}^{(1)}(N-1), \ldots, u_{i}^{(K)}(N-1)\right]^{T} .
\end{gathered}
$$

Then, from (10), we have

$$
\mathbf{x}_{i}=\overline{\mathbf{H B D}} \mathbf{u}_{i}+\boldsymbol{\eta}_{i}, \quad i=0,1, \ldots
$$

From (14), we obtain a least-square (LS) estimation as

$$
\mathbf{B D}_{i} \mathbf{u}_{i}=\left(\overline{\mathbf{H}}^{\dagger} \overline{\mathbf{H}}\right)^{-1} \overline{\mathbf{H}}^{\dagger} \mathbf{x}_{i} .
$$

Let $\mathbf{y}_{i}=\left(\overline{\mathbf{H}}^{\dagger} \overline{\mathbf{H}}\right)^{-1} \overline{\mathbf{H}}^{\dagger} \mathbf{x}_{i}$. Sectioning the vector into subvectors of length $K$ as

$$
\begin{aligned}
\mathbf{y}_{i} & =\left[\mathbf{y}_{i}^{T}(0), \mathbf{y}_{i}^{T}(1), \ldots, \mathbf{y}_{i}^{T}(N-1)\right]^{T} \\
\mathbf{u}_{i} & =\left[\mathbf{u}_{i}^{T}(0), \mathbf{u}_{i}^{T}(1), \ldots, \mathbf{u}_{i}^{T}(N-1)\right]^{T}
\end{aligned}
$$

we obtain

$$
\mathbf{y}_{i}(n)=\mathbf{b D}_{i}(n) \mathbf{u}_{i}(n)+\boldsymbol{\zeta}_{i}(n), \quad n=0,1, \ldots, N-1
$$

where $\boldsymbol{\zeta}_{i}(n)$ is the noise term. The $K \times K$ ambiguity matrix $\mathbf{b}$ and the diagonal matrices $\mathbf{D}_{i}(n)$ (only related to the $K$ CFOs) still need to be resolved. However, (17) is substantially simpler than (10) or (2), because the former dose not include the $J K$ multipath channel responses.

\section{Pilot Design for Resolving the CFOS AND AMBIGUITY}

We consider estimating the $K \times K$ ambiguity matrix $\mathbf{b}$ and the $K$ CFOs based on (17). Assume that a pilot block $\mathbf{u}_{i}$ is transmitted. We use specially designed pilots to simplify the computation. Two types of pilots are proposed. In the following, for simplicity of writing, the error terms associated with the noise are omitted in all equations.

\section{A. Periodical Pilots}

Let the time-domain pilot block be

$$
\begin{aligned}
\mathbf{u}_{i}(n) & =\alpha(n) \mathbf{e}(n), \quad n=0,1, \ldots, K-1 \\
\mathbf{u}_{i}(n+r K) & =\mathbf{u}_{i}(n), \quad r=0,1, \ldots
\end{aligned}
$$

where $\mathbf{e}(n)$ is a vector of length $K$, whose $(n+1)$ th entry is 1 and other entries are zeros, and $\alpha(n)$ is an arbitrary complex number. Table I shows the pilot pattern for $N=16$ and $K=4$.

Based on the periodical property of the pilots, we have

$$
\begin{aligned}
\mathbf{y}_{i}(n+r K) & =\mathbf{b D}_{i}(n+r K) \mathbf{u}_{i}(n) \\
& =\alpha(n) \mathbf{b D}_{i}(n+r K) \mathbf{e}(n)
\end{aligned}
$$

TABLE I

Periodical Pilot Pattern $(N=16, K=4)$

\begin{tabular}{|c|c|c|c|c|c|c|c|c|c|c|c|c|c|c|c|c|}
\hline user & \multicolumn{10}{|c|}{ pilot block in time domain } \\
\hline 1 & 1 & 0 & 0 & 0 & 1 & 0 & 0 & 0 & 1 & 0 & 0 & 0 & 1 & 0 & 0 & 0 \\
\hline 2 & 0 & 1 & 0 & 0 & 0 & 1 & 0 & 0 & 0 & 1 & 0 & 0 & 0 & 1 & 0 & 0 \\
\hline 3 & 0 & 0 & 1 & 0 & 0 & 0 & 1 & 0 & 0 & 0 & 1 & 0 & 0 & 0 & 1 & 0 \\
\hline 4 & 0 & 0 & 0 & 1 & 0 & 0 & 0 & 1 & 0 & 0 & 0 & 1 & 0 & 0 & 0 & 1 \\
\hline
\end{tabular}

$$
\begin{aligned}
& =\alpha(n) \mathbf{b}_{n} \omega_{N}^{f^{(n+1)}(n+r K+i M),} \\
n & =0,1, \cdots, K-1,
\end{aligned}
$$

where $\mathbf{b}_{n}$ is the $(n+1)$ th column of the matrix $\mathbf{b}$. Therefore

$$
\mathbf{y}_{i}^{\dagger}(n+m K) \mathbf{y}_{i}(n+(r+m) K)=|\alpha(n)|^{2}|| \mathbf{b}_{n} \|^{2} \omega_{P}^{f^{(n+1)} r},
$$

$m=0,1, \ldots, P-1-r$

where $P=\frac{N}{K}$. Let

$$
\begin{aligned}
\phi_{n}(r) & =\frac{1}{P-r} \sum_{m=0}^{P-1-r} \mathbf{y}_{i}^{\dagger}(n+m K) \mathbf{y}_{i}(n+(r+m) K) \\
& =|\alpha(n)|^{2}|| \mathbf{b}_{n} \|^{2} \omega_{P}^{f^{(n+1)} r},
\end{aligned}
$$

$$
r=0,1, \ldots, P-1 .
$$

Notice that $\mathbf{b}_{n} \neq 0$ because $\mathbf{b}$ is invertible. If the CFOs satisfy $\left|f^{(n+1)}\right|<P / 2$, they can be estimated from $\phi_{n}(1)$ as

$$
\tilde{f}^{(n+1)}=\frac{P}{2 \pi} \arg \left(\phi_{n}(1)\right), \quad n=0,1, \ldots, K-1
$$

where arg means the argument of a complex number. Here, we assume that $N \geq 2 K$.

We call this the coarse estimation. This estimation may not be very accurate. We can use it as an initial value to obtain a more accurate estimation by incorporating the contributions of all $\phi_{n}(r)$.

Let $\delta_{n}$ be the error of $\tilde{f}^{(n+1)}$, that is, $f^{(n+1)}=\tilde{f}^{(n+1)}+\delta_{n}$. If the coarse estimation satisfies the mild condition

$$
\left|f^{(n+1)}-\tilde{f}^{(n+1)}\right|<1
$$

then, $\left|\delta_{n}\right|<1$. Therefore

$$
\left|\frac{2 \pi r \delta_{n}}{P}\right|<\pi, \quad r=1,2, \ldots, \frac{P}{2} .
$$

From (21), we have

$$
\frac{2 \pi r \delta_{n}}{P}=\arg \left(\phi_{n}(r) \omega_{P}^{-\tilde{f}^{(n+1)} r}\right), \quad r=1,2, \ldots, \frac{P}{2} .
$$

An LS estimation for $\delta_{n}$ by using these $P / 2$ terms is then obtained as

$$
\tilde{\delta}_{n}=\frac{12}{\pi(P+1)(P+2)} \sum_{r=1}^{P / 2} r \arg \left(\phi_{n}(r) \omega_{P}^{-\tilde{f}^{(n+1)} r}\right) .
$$


We call $\hat{f}^{(n+1)}=\tilde{f}^{(n+1)}+\tilde{\delta}_{n}$ the two-step (TS) estimation for $f^{(n+1)}$.

In [5], a best linear unbiased estimator (BLUE) (the linear unbiased estimator having the MSE) is derived for the estimation of CFO in SISO-OFDM systems. The method can be borrowed to estimate the $f^{(n+1)}$ based on (21) (note that the noise term is omited). Let

$\rho_{n}(r)=\left[\arg \left(\phi_{n}(r)\right)-\arg \left(\phi_{n}(r-1)\right)\right]_{2 \pi}, \quad r=1,2, \ldots, P / 2$

where $[x]_{2 \pi}$ means the modulo- $2 \pi$ operation (it reduces $x$ to the interval $[-\pi, \pi))$. It can be verified that [5]

$$
\rho_{n}(r)=2 \pi f^{(n+1)} / P+\epsilon_{n}(r), \quad r=1,2, \ldots, P / 2
$$

where $\epsilon_{n}(r)$ is associated with noise. Based on (28) and similar derivations used in [5], the BLUE for $f^{(n+1)}$ is

$$
\begin{aligned}
\bar{f}^{(n+1)}= & \frac{3}{\pi\left(P^{2}-1\right)} \\
& \left.\cdot \sum_{r=1}^{P / 2}\left((P-r)(P-r+1)-P^{2} / 4\right)\right) \rho_{n}(r) .
\end{aligned}
$$

Remark: To obtain the BLUE, we need the statistical covariance matrix of $\left[\epsilon_{n}(1), \ldots, \epsilon_{n}(P / 2)\right]^{T}$. The covariance matrix here is very complicated. To simplify the problem, we have actually used the white noise assumption for $\boldsymbol{\zeta}_{i}(n)$, and an approximation similar to [5, eq. (9)], which is valid at high SNR but may not be accurate at low SNR. Usually, $\boldsymbol{\zeta}_{i}(n)$ is not white even if the ambient noise $\eta_{i}^{(j)}(n)$ is white. So, the BLUE may not always be the best. On the other hand, the TS estimator is dependent on the coarse estimation. If the coarse estimation satisfies the condition (23), the TS is the LS estimator, which does not rely on the white noise assumption. Ideally, if the SOS of the omitted noise term in (25) can be obtained, we can also use the MMSE estimator. Since the noise term is very complicated, we choose not to discuss the MMSE estimator here. The TS estimator has another advantage. If a coarse estimation for $f^{(n+1)}$ is known, no matter how large it is (it can be beyond the interval $[-N / 2 K, N / 2 K)$ ), if and only if it satisfies (23), we can use the aforementioned TS method to obtain a fine estimation. For example, in burst transmission mode, at the beginning of each burst, we can place more pilot symbols to obtain a full-range estimation for $f^{(n+1)}$, and then, insert the aforementioned pilot blocks periodically in the burst to track the CFO and the channel. Hence, the estimation range limitation can be overcome.

After the CFOs have been found, it is easy to obtain the ambiguity matrix $\mathbf{b}$. In fact, the $(n+1)$ th column of the matrix is estimated by

$$
\begin{aligned}
\mathbf{b}_{n} & =\frac{1}{P \alpha(n)} \sum_{r=0}^{P-1}\left[\mathbf{y}_{i}(n+r K) \omega_{N}^{-f^{(n+1)}(n+r K+i M)}\right], \\
n & =0,1, \ldots, K-1 .
\end{aligned}
$$

TABLE II

Consecutive Pilot Pattern ( $N=16, K=4)$

\begin{tabular}{|c|c|c|c|c|c|c|c|c|c|c|c|c|c|c|c|c|}
\hline user & \multicolumn{10}{|c|}{ pilot block in time domain } \\
\hline 1 & 1 & 1 & 1 & 1 & 0 & 0 & 0 & 0 & 0 & 0 & 0 & 0 & 0 & 0 & 0 & 0 \\
\hline 2 & 0 & 0 & 0 & 0 & 1 & 1 & 1 & 1 & 0 & 0 & 0 & 0 & 0 & 0 & 0 & 0 \\
\hline 3 & 0 & 0 & 0 & 0 & 0 & 0 & 0 & 0 & 1 & 1 & 1 & 1 & 0 & 0 & 0 & 0 \\
\hline 4 & 0 & 0 & 0 & 0 & 0 & 0 & 0 & 0 & 0 & 0 & 0 & 0 & 1 & 1 & 1 & 1 \\
\hline
\end{tabular}

Having known the ambiguity matrix $\mathbf{b}$, we obtain the modified channels (with CFOs) $\hat{\mathbf{h}}(l)=\overline{\mathbf{h}}(l) \mathbf{b}$. The real channels without CFOs are then found by depriving the CFOs from $\hat{\mathbf{h}}(l)$.

A problem for this design is that the estimation range is limited in $[-N / 2 K, N / 2 K)$. This problem will be resolved by using the consecutive pilots in the following.

\section{B. Consecutive Pilots}

Let the pilot block be

$$
\begin{aligned}
\mathbf{u}_{i}(l P+n) & =\alpha(l) \mathbf{e}(l), \\
l & =0,1, \ldots, K-1, n=0,1, \ldots, P-1 .
\end{aligned}
$$

Table II shows the pilot pattern for $N=16$ and $K=4$.

Based on the properties of the pilots, we have

$$
\begin{aligned}
\mathbf{y}_{i}(l P+n) & =\mathbf{b D}_{i}(l P+n) \mathbf{u}_{i}(l P+n) \\
& =\alpha(l) \mathbf{b} \mathbf{D}_{i}(l P+n) \mathbf{e}(l) \\
& =\alpha(l) \mathbf{b}_{l} \omega_{N}^{f^{(l+1)}(l P+n+i M)}, \\
l & =0,1, \ldots, K-1 .
\end{aligned}
$$

Therefore,

$$
\mathbf{y}_{i}^{\dagger}(l P+m) \mathbf{y}_{i}(l P+n+m)=|\alpha(l)|^{2}\left\|\mathbf{b}_{l}\right\|^{2} \omega_{N}^{f^{(l+1)} n} .
$$

Let

$$
\begin{aligned}
\psi_{l}(n) & =\frac{1}{P-n} \sum_{m=0}^{P-1-n} \mathbf{y}_{i}^{\dagger}(l P+m) \mathbf{y}_{i}(l P+n+m) \\
& =|\alpha(l)|^{2}\left\|\mathbf{b}_{l}\right\|^{2} \omega_{N}^{f^{(l+1)} n} \\
n & =0,1, \ldots, P-1 .
\end{aligned}
$$

Thus, the CFOs can be estimated from $\psi_{l}(1)$ as

$$
\tilde{f}^{(l+1)}=\frac{N}{2 \pi} \arg \left(\psi_{l}(1)\right), l=0,1, \ldots, K-1 .
$$

Although (35) gives a full-range estimation for the CFOs, it may not be accurate enough due to the noise, and statistic and roundoff errors. We call this the coarse estimation. Similar as before, we can use it as an initial value to obtain a more accurate estimation. Let $f^{(l+1)}=\tilde{f}^{(l+1)}+\delta_{l}$. If the error term satisfies

$$
\left|\delta_{l}\right|<\frac{N}{P}=K
$$

then

$$
\left|\frac{2 \pi n \delta_{l}}{N}\right|<\pi, n=1,2, \ldots, \frac{P}{2} .
$$


Therefore, from (34), we have

$$
\frac{2 \pi n \delta_{l}}{N}=\arg \left(\psi_{l}(n) \omega_{N}^{-\tilde{f}^{(l+1)} n}\right), n=1,2, \ldots, \frac{P}{2} .
$$

An LS estimation for $\delta_{l}$ is then obtained as

$$
\tilde{\delta}_{l}=\frac{12 K}{\pi(P+1)(P+2)} \sum_{n=1}^{P / 2} n \arg \left(\psi_{l}(n) \omega_{N}^{-\tilde{f}^{(l+1)} n}\right) .
$$

We call $\hat{f}^{(l+1)}=\tilde{f}^{(l+1)}+\tilde{\delta}_{l}$ the TS estimation for $f^{(l+1)}$.

Similarly, based on some approximations, the BLUE for $f^{(l+1)}$ can be obtained. Let

$$
\varrho_{l}(n)=\left[\arg \left(\psi_{l}(n)\right)-\arg \left(\psi_{l}(n-1)\right)\right]_{2 \pi}, n=1,2, \ldots, \frac{P}{2} .
$$

The BLUE for $f^{(l+1)}$ is

$$
\begin{aligned}
\bar{f}^{(l+1)}= & \frac{3 K}{\pi\left(P^{2}-3 N P+3 N^{2}-1\right)} \\
& \left.\cdot \sum_{n=1}^{P / 2}((N-n)(N-n+1)-P(2 N-P) / 4)\right) \varrho_{l}(n) .
\end{aligned}
$$

Similar as before, the $(l+1)$ th column of the ambiguity matrix $\mathbf{b}$ is estimated by

$$
\begin{aligned}
\mathbf{b}_{l} & =\frac{1}{P \alpha(l)} \sum_{n=0}^{P-1}\left[\mathbf{y}_{i}(l P+n) \omega_{N}^{-f^{(l+1)}(l P+n+i M)}\right], \\
l & =0,1, \ldots, K-1 .
\end{aligned}
$$

Here, the estimation range limit is $[-N / 2, N / 2)$, which is the largest possible estimation range for the CFOs.

\section{Signal Recovery}

When the CFOs have been estimated at the base station, recovery of the signals is still a problem. The CFOs destroy the orthogonality among the subcarriers and introduce the ICI. Therefore, the "per-tone" equalization cannot be used anymore. For an SISO-OFDM, it is easy to compensate the CFO by the receiver and then use the "per-tone" equalization. However, this is invalid for MIMO-OFDM with multiple different CFOs. In the following, we will give a method to recover the signals based on the FFT. The computational complexity is only moderately higher than that of the "per-tone" equalization [1].

As in [1], each received block is overlap-added as

$$
\hat{x}_{i}^{(j)}(n)=\left\{\begin{array}{lr}
x_{i}^{(j)}(n)+x_{i}^{(j)}(n+N), & n=0, \ldots, L-1 \\
x_{i}^{(j)}(n), & n=L, \ldots, N-1 .
\end{array}\right.
$$

Each overlap-added block is then transformed by the DFT. Let $\hat{X}_{i}^{(j)}(n), \hat{H}^{(j, k)}(n)$, and $\hat{U}_{i}^{(k)}(n)$ be the DFT of $\hat{x}_{i}^{(j)}(n)$, $\hat{h}^{(j, k)}(n)$, and $\hat{u}_{i}^{(k)}(n)$, respectively. Then, from (4), we have

$$
\begin{aligned}
\hat{X}_{i}^{(j)}(n) & =\sum_{k=1}^{K} \hat{H}^{(j, k)}(n) \hat{U}_{i}^{(k)}(n)+\boldsymbol{\xi}_{i}^{(j)}(n), \\
n & =0,1, \ldots, N-1
\end{aligned}
$$

where $\boldsymbol{\xi}_{i}^{(j)}(n)$ is the noise after the DFT. Defining

$$
\begin{aligned}
\hat{\mathbf{X}}_{i}(n) & =\left[\hat{X}_{i}^{(1)}(n) \cdots \hat{X}_{i}^{(J)}(n)\right]^{T} \\
\hat{\mathbf{U}}_{i}(n) & =\left[\hat{U}_{i}^{(1)}(n) \cdots \hat{U}_{i}^{(K)}(n)\right]^{T} \\
\boldsymbol{\xi}_{i}(n) & =\left[\xi_{i}^{(1)}(n) \cdots \xi_{i}^{(J)}(n)\right]^{T}
\end{aligned}
$$

$$
\hat{\mathbf{H}}(n)=\left[\begin{array}{cccc}
\hat{H}^{(1,1)}(n) & \cdots & \cdots & \hat{H}^{(1, K)}(n) \\
\hat{H}^{(2,1)}(n) & \cdots & \cdots & \hat{H}^{(2, K)}(n) \\
\vdots & \vdots & & \vdots \\
\hat{H}^{(J, 1)}(n) & \cdots & \cdots & \hat{H}^{(J, K)}(n)
\end{array}\right]
$$

we obtain

$$
\hat{\mathbf{X}}_{i}(n)=\hat{\mathbf{H}}(n) \hat{\mathbf{U}}_{i}(n)+\boldsymbol{\xi}_{i}(n) .
$$

Thus, $\hat{U}_{i}^{(k)}(n)$ can be recovered per-tonely by the LS method as

$$
\hat{\mathbf{U}}_{i}(n)=\left(\hat{\mathbf{H}}^{\dagger}(n) \hat{\mathbf{H}}(n)\right)^{-1} \hat{\mathbf{H}}^{\dagger}(n) \hat{\mathbf{X}}_{i}(n) .
$$

If no CFOs, that is, $f^{(k)}=0$, then $\hat{U}_{i}^{(k)}(n)=s_{i}^{(k)}(n)$ and the signals are obtained. When CFOs exist, we implement the IDFT on $\hat{U}_{i}^{(k)}(n)$ to obtain $\hat{u}_{i}^{(k)}(n)$. From (3), the transmitted time-domain signals without CFOs are found as

$$
u_{i}^{(k)}(n)=\omega_{N}^{-f^{(k)}(n+i M)} \hat{u}_{i}^{(k)}(n) .
$$

Finally, we get the transmitted frequency-domain signal $s_{i}^{(k)}(n)$ by implementing the DFT on $u_{i}^{(k)}(n)$.

Compared with the equalization method for the MIMOOFDM without CFOs, here the computational complexity only increases by two FFTs for each user. The order of the computational complexities remains at $\mathrm{O}\left((J+K) N \log _{2} N\right)$ (multiplications and additions).

\section{Simulations}

In the following, SNR means the ratio of the average received signal power to the average noise power as

$$
\mathrm{SNR}=\mathrm{E}\left(\left\|\mathbf{x}_{i}-\boldsymbol{\eta}_{i}\right\|^{2}\right) / \mathrm{E}\left(\left\|\boldsymbol{\eta}_{i}\right\|^{2}\right) .
$$

The normalized MSE (NMSE) between the estimated and the true channel responses is defined as

$$
\operatorname{NMSE}(\text { for channel })=\frac{\sum_{l=1}^{L}\left\|\mathbf{h}(l)-\mathbf{h}^{\prime}(l)\right\|_{F}^{2}}{\sum_{l=1}^{L}\|\mathbf{h}(l)\|_{F}^{2}}
$$

where $\mathbf{h}^{\prime}(l)$ and $\mathbf{h}(l)$ are the estimated and the true channel matrices, respectively, and $\|\cdot\|_{F}$ means the Frobenius norm of matrix. Similarly, the NMSE between the estimated and the true CFOs is defined as

$$
\mathrm{NMSE}(\text { for } \mathrm{CFO})=\frac{\sum_{k=1}^{K}\left|f^{(k)}-f^{\prime(k)}\right|^{2}}{\sum_{k=1}^{K}\left|f^{(k)}\right|^{2}}
$$

where $f^{(k)}$ and $f^{\prime(k)}$ are the true and estimated CFO of user $k$, respectively. 


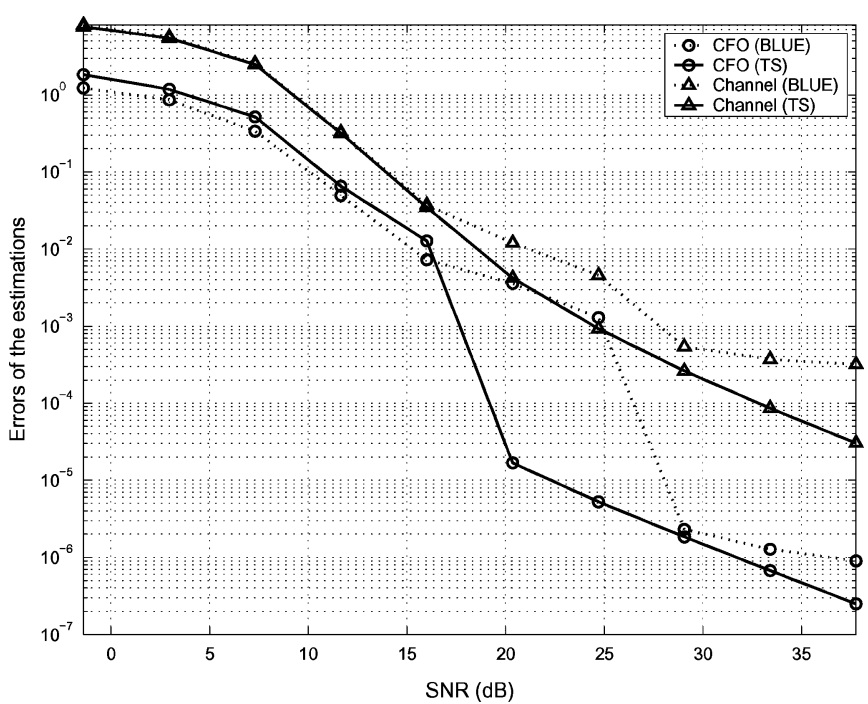

Fig. 2. NMSEs versus SNRs (periodical pilots).

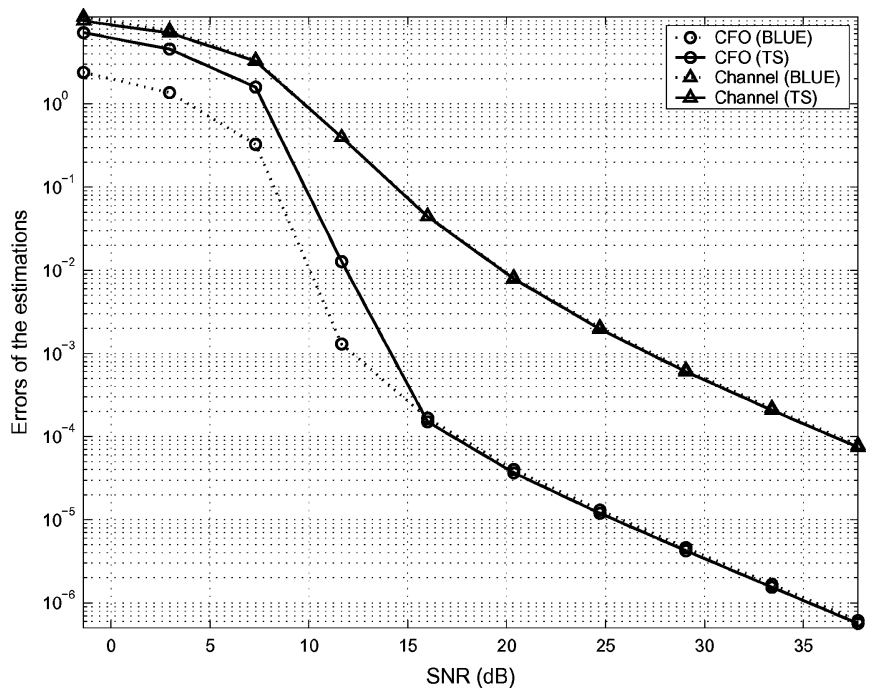

Fig. 3. NMSEs versus SNRs (consecutive pilots).

We consider a 2-user and 3-antenna MIMO system. The system parameters are: 1) the maximum length of discrete channels is $9 ; 2$ ) the transmitted baseband signals are 4-QAM; and 3) the length of each OFDM block is $N=32$, which is zero-padded to block of length $M=40$. We assume that the first transmitted OFDM block of each user is the specially designed pilot block. For computing the SOS needed for the subspace method in [1], 150 received blocks are used.

1) NMSEs for CFO and Channel Estimations: The NMSEs defined earlier are used to measure the accuracy of CFO estimation and channel estimation, respectively. Fig. 2 shows the results when the periodical pilots are used. Figs. 3 and 4 show the errors of estimations when the consecutive pilots are used. For Figs. 2 and 3, the CFOs are evenly distributed in $[-N / 2 K, N / 2 K)$, and for Fig. 4, CFOs are evenly distributed in $[-N / 2, N / 2)$. All the results, here, are averaged over 1000 Monte Carlo realizations (for each realization, random evenly distributed CFOs, random

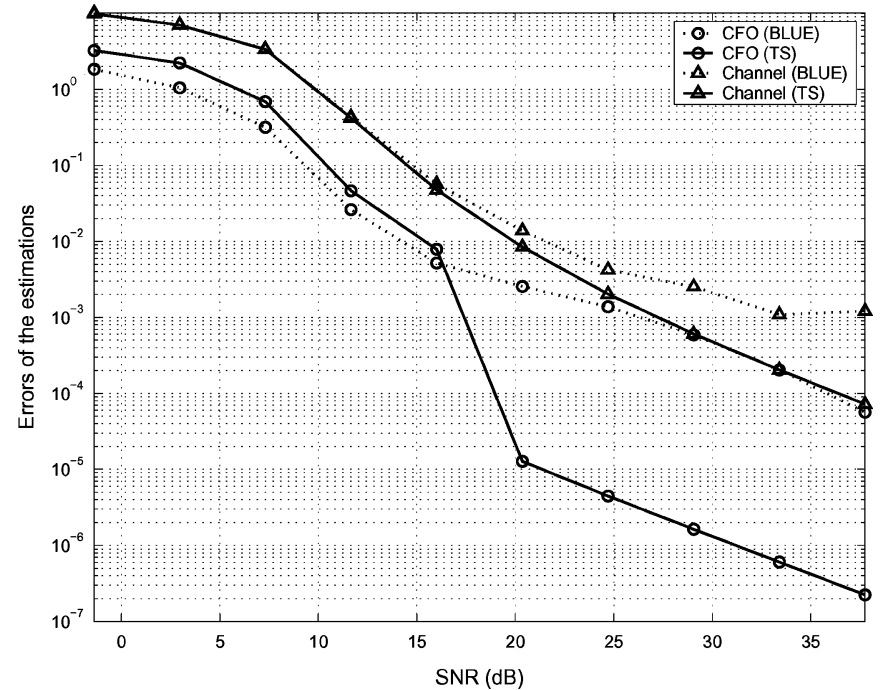

Fig. 4. NMSEs versus SNRs (consecutive pilots, full range CFOs).

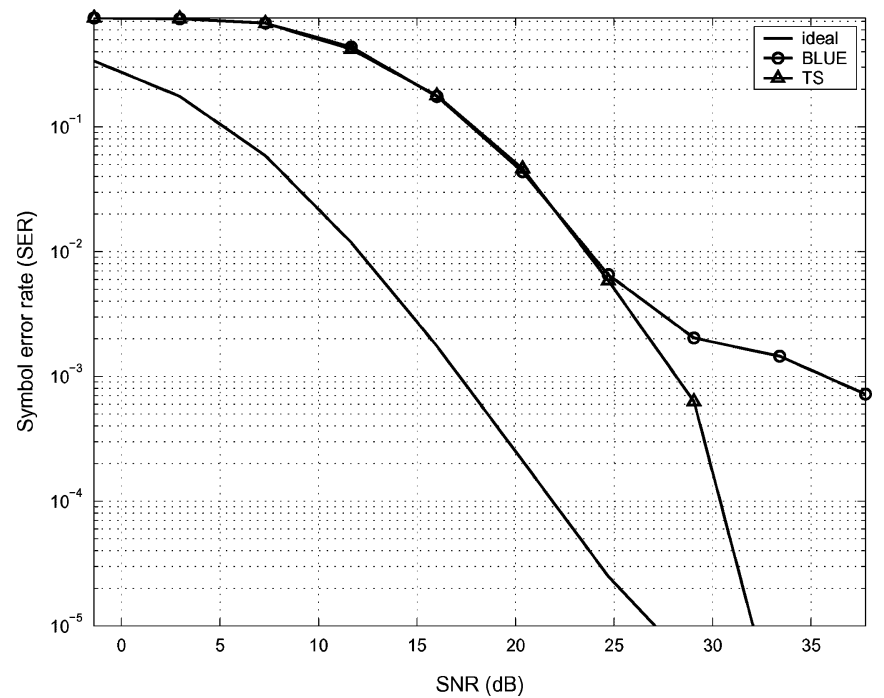

Fig. 5. SERs versus SNRs (periodical pilots).

Rayleigh fading channels with 9 taps, random inputs, and random noises are used). The results show that the TS is generally better than the BLUE, especially when the CFOs approach the end of the largest estimation interval (the largest estimation intervals for periodical and consecutive pilots are $[-N / 2 K, N / 2 K)$ and $[-N / 2, N / 2)$, respectively). In Fig. 3, since the CFOs are within a small subinterval of the largest estimation interval, the two methods have nearly the same performance. Comparing Figs. 2 and 3 , we see that using the periodical pilots is better if the CFOs are limited in $[-N / 2 K, N / 2 K)$. As shown in Fig. 4, the advantage of consecutive pilots is that it can be used for estimating the CFOs of full range.

2) Symbol-Error Rates (SERs) for Signal Recovery: Looking at (2) or (51), we see that the SER is very sensitive to CFO estimation errors, if the time $n+i M$ is large. For example, if the NMSE of the CFO is around $10^{-6}$ and $n+i M$ 


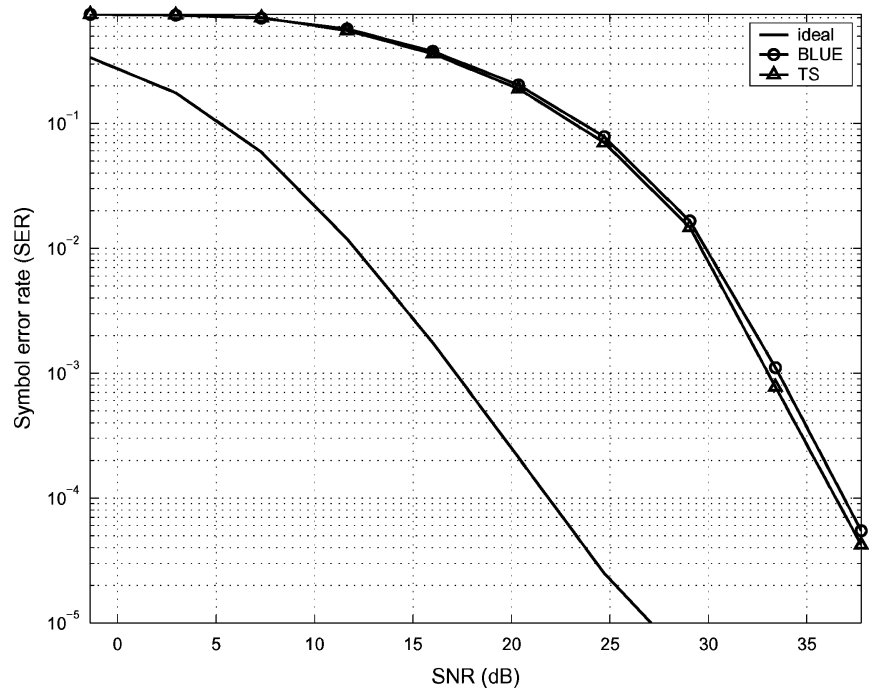

Fig. 6. SERs versus SNRs (consecutive pilots).

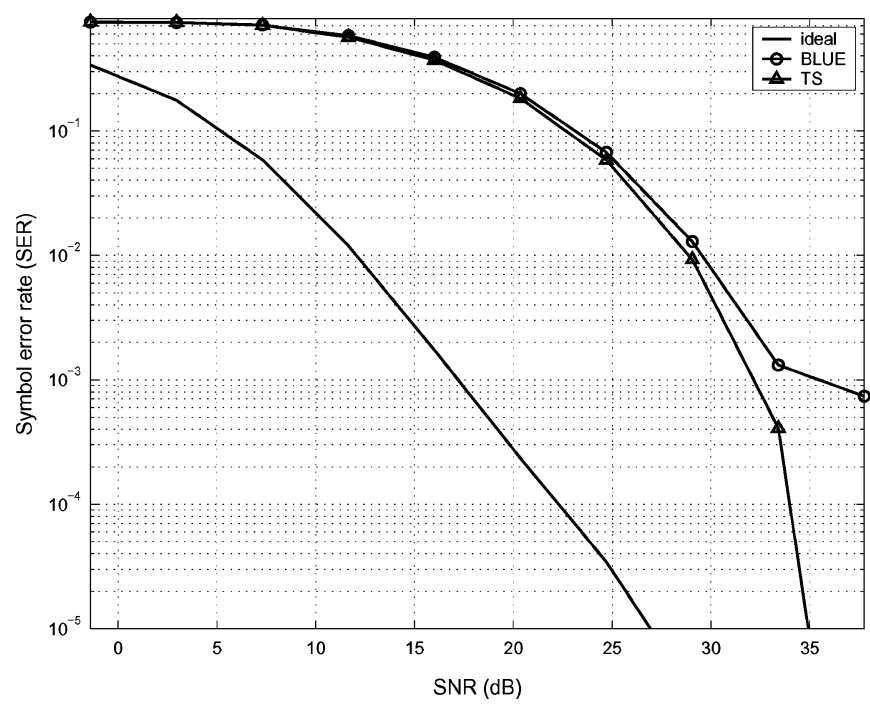

Fig. 7. SERs versus SNRs (consecutive pilots, full-range CFOs).

$=1000$, then, from (51), the CFO error will approximately produce an error factor $\omega_{N}^{-1}=e^{-2 \pi / N}$ for the symbols. So, in practice, we must reset the timing after a short period of time. In the following, we assume that the timing is reset after 10 OFDM blocks. Figs. 5-7 show the SERs, where the settings are the same as those in Figs. 2-4, respectively. The curve without marks is for the "ideal" case: real channels and real CFOs are used. The results are averaged over 20000 OFDM blocks. It is obvious that the TS method generally gives better SERs than the BLUE does. The results also reassure the findings declared earlier.

\section{CONCLUSION}

An efficient semiblind method has been proposed for jointly estimating the CFOs and channels of an uplink multiuser MIMO-OFDM system. Based on the SOS of the received signal and one specially designed pilot OFDM block for each user, the CFOs and channels are found simultaneously. Furthermore, a fast equalization method has been given to recover the signals corrupted by the CFOs.

\section{ACKNOWLEDGMENT}

The authors would like to thank the Editor and the anonymous reviewers for their invaluable comments.

\section{REFERENCES}

[1] Y. H. Zeng and T. S. Ng, "A semi-blind channel estimation method for multi-user multi-antenna OFDM systems," IEEE Trans. Signal Process., vol. 52, no. 5, pp. 1419-1429, May 2004.

[2] H. Sampath, S. Talwar, J. Tellado, V. Erceg, and A. Paulraj, "A fourthgeneration MIMO-OFDM braodband wireless system: Design, performance, and field trial results," IEEE Commun. Mag., vol. 40, no. 9, pp. 143-149, Sep. 2002.

[3] Y. W. Yao and G. B. Giannakis, "Blind carrier frequency offset estimation in SISO, MIMO, and multiuser OFDM systems," IEEE Trans. Commun., vol. 53, no. 1, pp. 173-183, Jan. 2005.

[4] J. Van de Beek, M. Sandell, and P. O. Börjesson, "ML estimation of time and frequency offset in OFDM systems," IEEE Trans. Signal Process., vol. 45 , no. 7 , pp. 1800-1805, Jul. 1997.

[5] M. Morelli and U. Mengali, "An improved frequency offset estimator for OFDM applications,” IEEE Commun. Lett., vol. 3, no. 3, pp. 75-77, Mar. 1999.

[6] H. K. Song, Y. H. You, J. H. Paik, and Y. S. Cho, "Frequency-offset synchronization and channel estimation for OFDM-based transmission," IEEE Commun. Lett., vol. 4, no. 3, pp. 95-97, Mar. 2000.

[7] U. Tureli, P. J. Honan, and H. Liu, "Low-complexity nonlinear least square carrier offset estimator for OFDM: Identifiability, diversity and performance," IEEE Trans. Signal Process., vol. 52, no. 9, pp. 2441-2452, Sep. 2004.

[8] X. Ma, G. B. Giannakis, and S. Barbarossa, "Non-data-aided frequencyoffset and channel estimation in OFDM and related block transmissions,' in Proc. IEEE ICC, 2001, pp. 1866-1870.

[9] X. Ma, C. Tepedelenlioglu, G. B. Giannakis, and S. Barbarossa, "Nondata-aided carrier offset estimators for OFDM with null subcarriers: Identifiability, algorithms, and performance," IEEE J. Sel. Areas Commun., vol. 19, no. 12, pp. 2504-2515, Dec. 2001.

[10] M. Ghogho, A. Swami, and G. B. Giannakis, "Optimized null-subcarrier selection for CFO estimation in OFDM over frequency selective fading channels," in Proc. IEEE GlobeCom, 2001, pp. 202-206.

[11] J. Lei and T. S. Ng, "A consistent OFDM carrier frequency offset estimator based on distinctively spaced pilot tones," IEEE Trans. Wireless Commun., vol. 3, no. 3, pp. 588-599, Mar. 2004.

[12] A. N. Mody and G. L. Stuber, "Synchronization for MIMO OFDM systems," in Proc. IEEE GlobeCom, 2001, pp. 509-513.

[13] A. V. Zelst and T. C. W. Schenk, "Implementation of a MIMO OFDMbased wireless LAN system,” IEEE Trans. Signal Process., vol. 52, no. 2, pp. 483-494, Feb. 2004

[14] Z. R. Cao, U. Tureli, and Y. D. Yao, "Deterministic multiuser carrierfrequency offset estimation for interleaved OFDMA uplink," IEEE Trans. Commun., vol. 52, no. 9, pp. 1585-1594, Sep. 2004.

[15] Y. Yao and T. S. Ng, "Correlation-based frequency offset estimation in MIMO system," in Proc. IEEE VTC FALL, Oct. 6-9, 2003, vol. 1, pp. 438442.

[16] O. Besson and P. Stoica, "On parameter estimation of MIMO flat-fading channels with frequency offsets," IEEE Trans. Signal Process., vol. 51, no. 3, pp. 602-613, Mar. 2003.

[17] H. Bölcskei, R. W. Heath Jr., and A. J. Paulraj, "Blind channel identification and equalization in OFDM-based multi-antenna systems," IEEE Trans. Signal Process., vol. 50, no. 1, pp. 96-109, Jan. 2002.

[18] Y. Li and H. Wang, "Channel estimation for MIMO-OFDM wireless communications," in Proc. 14th IEEE 2003 Int. Symp. Pers., Indoor Mobile Radio Commun., pp. 2891-2895.

[19] Y. H. Zeng, A. R. Leyman, S. D. Ma, and T. S. Ng, "Optimal pilot and fas algorithm for MIMO-OFDM channel estimation," presented at the Int. Conf. Inf. Commun. Signal Process. (ICICS), Bangkok, Thailand, Dec. 2005. 
[20] I. B. Barhumi, G. Leus, and M. Moonen, "Optimal training design for MIMO OFDM systems in mobile wireless channels," IEEE Trans. Signal Process., vol. 51, no. 6, pp. 1615-1624, Jun. 2003.

[21] G. J. Foschini, "A layered space-time architecture for wireless communication in a fading environment using multi-element antennas," Bell Labs. Tech. J., vol. 1, no. 2, pp. 41-59, 1996.

[22] P. W. Wolniansky, G. J. Foschini, G. D. Golden, and R. A. Valenzuela, "V-BLAST: An architecture for realizing very high data rates over the rich-scattering wireless channels," in Proc. URSI Int. Symp. Signals, Syst., Electron. (ISSSE), 1998, pp. 295-300.

[23] A. Sklavos, T. Weber, E. Costa, H. Haas, and E. Schulz, "Joint detection in multi-antenna and multi-user OFDM systems," in Multi-Carrier SpreadSpectrum and Related Topics, K. Fazel and S. Kaiser, Eds. Boston, MA: Kluwer, 2002.

[24] Z. R. Cao, U. Tureli, Y. D. Yao, and P. Honan, "Frequency synchronization for generalized OFDMA uplink," in Proc. IEEE Globecom, 2004 pp. 1071-1075.

[25] A. Scaglione, G. B. Giannakis, and S. Barbarossa, "Redundant filterbank precoders and equalizers, Part II: Blind channel estimation, synchronization and direct equalization," IEEE Trans. Signal Process., vol. 47, no. 7, pp. 2007-2022, Jul. 1999

[26] G. B. Giannakis, Y. Hua, P. Stoica, and L. Tong, Signal Processing Advances in Wireless and Mobile Communications, vol. 1. Englewood Cliffs, NJ: Prentice-Hall, 2001.

[27] B. Muquet, Z. Wang, G. B. Giannakis, M. D. Courville, and P. Duhamel, "Cyclic prefixing or zero padding for wireless multicarrier transmissions," IEEE Trans. Commun., vol. 50, no. 12, pp. 2136-2148, Dec. 2002.

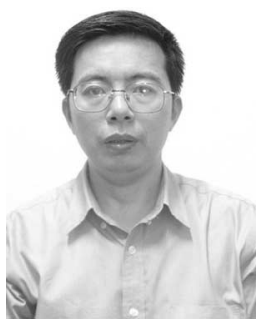

Yonghong Zeng (A'00-M'01-SM'05) received the B.S. degree from Peking University, Beijing, China, in 1983, and the M.S. and Ph.D. degrees from the National University of Defense Technology, Changsha, China, in 1986 and 1998, respectively.

Prior to July 1999, he worked as an Associate Professor with the National University of Defense Technology. From August 1999 to October 2004, he was a Research Fellow at the Nanyang Technological University, Singapore, and the University of Hong Kong, Hong Kong, successively. Since November 2004, he has been a Scientist with the Institute for Infocomm Research, Agency for Science, Technology and Research (A*STAR), Singapore. His current research interests include signal processing and wireless communication, especially on cognitive radio and software-defined radio, channel estimation, equalization, detection, and synchronization. He is the author or coauthor of more than 60 refereed journal papers and six books, including the Transforms and Fast Algorithms for Signal Analysis and Representation (Springer-Birkhuser, Boston, 2003).

Dr. Zeng is the four-time recipient of the Ministry-level Scientific and Technological Development Award in China.

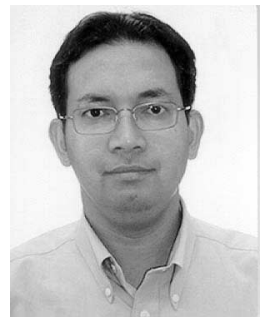

A. Rahim Leyman received the Ph.D. degree in electrical engineering from the University of Strathclyde, Glasgow, U.K., in 1994.

From 1995 to 2001, he was an Assistant Professor at the Nanyang Technological University, Singapore. Since 2001, he is a Senior Scientist at the Institute for Infocomm Research (I2R), Agency for Science, Technology, and Research (A*STAR), Singapore. He is currently an Adjunct Associate Professor in the Department of Electrical and Computer Engineering, National University of Singapore, Singapore. His current research interests include applications of statistical signal processing to communication and biomedical signal processing, blind source separation, sensor array processing, higher order statistics, and non-Gaussian signal processing.

Dr. Leyman was the Co-Chairman for the IEEE Signal Processing Society Workshop on Statistical Signal Processing, 2001 publishes widely in IEEE TRANSACTIONS ON Signal PROCESSING (TSP) and is currently an Associate Editor of the TSP. He is an active Reviewer for IEEE SIGNAL PROCESSING LETTERS, IEEE TRANSACTIONS ON SIGNAL PROCESSING, IEEE COMMUNICATIONS LETTERS, and IEEE TRANSACTIONS ON COMMUNICATIONS, and the International Conference Axoustics, Speech, and Signal Processing (ICASSP) conferences.

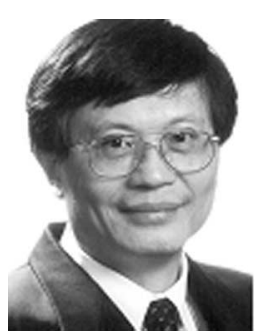

Tung-Sang Ng (S'74-M'78-SM'90-F'03) received the B.Sc. (Eng.) degree from the University of Hong Kong, Kowloon Tong, Hong kong, in 1972, and the M.Eng.Sc. and Ph.D. degrees from the University of Newcastle, Newcastle, Australia, in 1974 and 1977 , respectively, all in electrical engineering.

He was with the Billiton (BHP) Steel International and the University of Wollongong, Australia for 14 years before returning to the University of Hong Kong, Hong Kong, in 1991, taking up the position of Professor and Chair of Electronic Engineering. From 2000 to 2003, he was the Head of the Department of Electrical and Electronic Engineering, University of Hong Kong, and is currently the Dean of Engineering. His current research interests include wireless communication systems, spread-spectrum techniques, code -division-multiple access (CDMA), and digital signal processing. He is the author or coauthor of than 250 international journals and conference papers.

Dr. T.-S. Ngwas is a Fellow of the the Institute of Electrical Engineers (IEE) and Hong Kong Institute of Engineers (HKIE). He was the General Chair of the 1997 International Symposium on Circuits and Systems (ISCAS'97) and the Vice-President-Region 10 of the IEEE Circuits and Systems (CAS) Society in 1999 and 2000. From 1999 to 2001, he was an Executive Committee Member and a Board Member of IEE Informatics Divisional Board, and also an ordinary member of the IEE Council. He was the recipient of the Honorary Doctor of Engineering Degree from the University of Newcastle in 1997, the Senior Croucher Foundation Fellowship Award in 1999, the IEEE Third Millenium medal in 2000, and the Outstanding Researcher Award from the University of Hong Kong in 2003. 